\title{
Die Löslichkeitsbedingungen von Calciumsalzen im Harn von Patienten mit Urolithiasis
}

\author{
von \\ Hans-Joachim Dulce und Eva Taupitz \\ Aus der Urologischen Universitätsklinik Homburg/Saar (Direktor: Prof. Dr. C.-E. Alken) \\ und aus dem Physiologisch-chemischen Institut der Freien Universität Berlin-Dablem (Direktor: Prof. Dr. Dr. E. Scbütte)
}

(Der Schriftleitung zugegangen am 8. Dezember 1962)

\begin{abstract}
Es wurden die Löslichkeitsbedingungen für Calciumoxalat und Calciumphosphat im 24 Stdn.-Harn bei 2 Gruppen von Steinkranken und einer Gruppe Gesunder untersucht. Bei der Hälfte der Steinkranken finden sich im Harn schlechtere Löslichkeitsbedingungen für Calciumoxalat oder Calciumphosphat als bei Gesunden. Eine Hauptvoraussetzung des Steinwachstums, die Kristallisationsbereitschaft des Harnes, ist damit erfüllt.
\end{abstract}

\begin{abstract}
The solubility of calcium oxalate and calcium phosphate was studied in 24-hour urine samples from two groups of patients with kidney stones and from a healthy group. Calcium oxalate or calcium phosphate were less soluble in one part of the "stone" urines than in healthy urine. The chief requirement for stone growth, i. e., tendency of the urine to deposit crystals, is therefore present.
\end{abstract}

Als Voraussetzungen der Harnsteinbildung betrachten wir heute einen Kristallisationskern und einen kristallisationsbereiten Harn. Eine Harnstauung kann zusätzlich durch Strömungsverlangsamung günstige Wachstumsbedingungen für Konkremente schaffen (1). Normaler Harn ist nicht kristallisationsbereit. Das zeigen fehlgeschlagene Versuche, durch Impfen des Harnes mit Kristallen Kristallwachstum zu erreichen (2). Das zeigen weiter Berechnungen und experimentelle Untersuchungen der Löslichkeit von Calciumsalzen im Harn $(3,4,5)$. Im Harn von Steinkranken müssen demnach kontinuierlich oder zeitlich begrenzt besonders ungünstige Löslichkeitsverhältnisse für steinbildende Substanzen vorliegen.

Die Löslichkeit von Calciumoxalat im Harn wird in erster Linie durch die $\mathrm{Ca}^{++}$- und Oxalationenausscheidung bestimmt. Je größer im Harn das Ionenprodukt gegenüber dem Löslichkeitsprodukt von Calciumoxalat ( $3 \times 10^{-9}$ Monohydrat, $7 \times 10^{-9}$ Dihydrat) ist, desto unlöslicher kann Calciumoxalat werden. In zweiter Linie hängt aber die Löslichkeit des Calciumoxalats auch von der Konzentration seiner Lösungsvermittler ab. Magnesiumionen, Fremdelektrolyte $(4,5)$, anorgan. und organ. Phosphate (6), und Citrat steigern die Löslichkeit des Calciumoxalats erheblich, weil sie die Calcium- oder Oxalationenaktivität herabsetzen. Die Löslichkeit der Calciumphosphate wird außer durch die Calcium- und Phosphatausscheidung hauptsächlich durch den $\mathrm{pH}$ Wert des Harnes bestimmt. Bei $\mathrm{pH}<6,5$ ist Calciumphosphat löslich, bei $\mathrm{pH}>6,5$ nimmt die Unlöslichkeit der Calciumphosphate stetig zu. In diesem pH-Bereich wird die Löslichkeit wiederum durch Magnesiumionen, Fremdelektrolyte (8) und bei $\mathrm{pH} 7-8$ auch durch Citrat (7) erhöht. Harnkolloide beeinflussen weder die Löslichkeit des Calciumoxalats noch des Calciumphosphats
$(5,8)$. Die Beziehungen zwischen der Konzentration von Calcium, Magnesium, Phosphat, Oxalsäure oder Kolloiden im Harn und der Art und dem Ausmaß von Steinerkrankungen des Harntraktes sind häufig untersucht worden. In der Regel wurde die Tagesausscheidung dieser Ionen der Diskussion zugrundegelegt. 35 von 199 untersuchten Patienten mit calciumhaltigen Steinen hatten eine Hypercalcurie von $300 \mathrm{mg} / \mathrm{Tag}$ und darüber (9). Eine Gruppe Gesunder schied nur bis $150 \mathrm{mg}$ Calcium/Tag bei gleicher Kost aus. Auch Cotret und Mitarbeiter (10) beobachteten bei Steinktanken häufiger Hypercalcurien als bei Gesunden. Bei rezidivierenden Steinerkrankungen auf infektiöser Basis fanden sie allerdings auch Hypocalcurien, die sie als Folge einer tubulären Nierenschädigung ansahen. BOYCE und Mitarbeiter (11) wiesen bei Patienten mit nicht wachsenden Steinen keine, bei solchen mit wachsenden Steinen eine deutliche Hypercalcurie nach. Eine erhöhte Tagesausscheidung an Phosphat scheint bei Phosphatsteinträgern nicht zu bestehen. Wohl aber sind weniger org. Phosphate im Harn von Steinkranken als im Harn von Gesunden nachgewiesen worden (6). Die Oxalatausscheidung wurde bei Oxalatsteinkranken in $20-30 \%$ der Fälle über $34 \mathrm{mg} / \mathrm{Tag}$ gefunden (normal $20-30 \mathrm{mg}$ / Tag) $(12,13,14)$. Dem steht gegenüber, daß SENGBUSCH und Mitarbeiter (15) mit der Siebmethode bei Oxalatsteinkranken in $80 \%$ der Fälle viel mittlere und große Calciumoxalatkristalle im frisch gelassenen Harn fanden. Das Calciumoxalat muß also nicht nur durch vermehrte Oxalatausscheidung unlöslicher werden. Eine im Verhältnis zum Calcium herabgesetzte Magnesiumausscheidung ist bisher nur bei wenigen Steinkranken bekannt geworden $(4,16,17)$. Wir wissen aber, daß in Ländern, in denen magnesiumreiche Kost üblich ist (Finnland, Ägypten), Harnsteine selten sind (4). Die Citrataus- 
scheidung wurde zwar von Kissin (18) bei Calciumoxalatsteinkranken oft erniedrigt gefunden $(30 \mathrm{mg} / \mathrm{Tag})$. TAUPITZ und GACA (19) konnten aber kürzlich zeigen, $\mathrm{da} B$ die Citratausscheidung bei vielen nephrotischen und pyelonephritischen Erkrankungen ohne Konkremente ebenfalls stark erniedrigt (unter $30 \mathrm{mg} / \mathrm{Tag}$ ) sein kann.

Mit diesen Befunden hat man versucht, eine ursächliche Beziehung $z$ wischen den Löslichkeitsbedingungen steinbildender Salze im Harn und der Urolithiasis zu begründen. Diese Folgerungen schienen uns nicht stichhaltig, weil man die Löslichkeit eines Salzes nur beurteilen kann, wenn die molaren Konzentrationen des beteiligten Kations und Anions gleichzeitig bekannt sind, man also das aktuelle Ionenprodukt mit dem Löslichkeitsprodukt vergleichen kann. Des weiteren gestatten nur die Konzentrationen lösungsvermittelnder Ionen in Beziehung zum aktuellen Ionenprodukt der konkrementbildenden Ionen Aussagen über bessere oder schlechtere Löslichkeit des unlöslichen Salzes. Wir bestimmten deshalb in der vorliegenden Arbeit bei Steinkranken gleichzeitig die Konzentration der Konkrementbildenden Ionen und ihrer wichtigsten Lösungsvermittler im Tagesharn. Außerdem ermittelten wir den Grad bakteriell entzündlicher Veränderungen im Harntrakt und die Ausscheidung der gesamten Harnkolloide, um Anhaltspunkte über den Einfluß bakterieller Umsetzungen im Harn zu gewinnen.

\section{Methodik}

Wir untersuchten 3 Gruppen von Personen, die eine durchschnittliche Krankenhauskost erhielten: a) $7 \mathrm{Ge}-$ sunde; b) 15 Patienten mit nachgewiesenen Calciumoxalatsteinen; c) 16 Patienten mit Ca-haltigen Rö-schattengebenden Steinen. Es wurde der 24-Stdn.-Harn an einem oder zwei aufeinanderfolgenden Tagen gesammelt und im Kühlschrank aufbewahrt. Ein aliquoter Teil des Harnes wurde im Vakuum eingedampft und der Rückstand im Exsikkator über Silicagel getrocknet. Im pulverisierten Trockenrückstand, dessen Gesamtgewicht bekannt war, führten wir folgende Untersuchungen, jeweils mit Doppelbestimmungen durch:

$1 \mathrm{~g}$ der Harntrockensubstanz wurde bei $600-650^{\circ}$ im Muffelofen verascht und der Rückstand quantitativ in $20 \mathrm{~m} / 2 n \mathrm{HCl}$ gelöst. Diese Lösung diente für folgende Bestimmungen:

1. Kalium und Natriumbestimmung: Wir verdünnten $1,0 \mathrm{ml}$ mit aq. dest. auf $100 \mathrm{ml}$ und bestimmten flammenphotometrisch.

2. Bestimmung des anorg. Phosphats (berechnet als anorg. P.): $2 \mathrm{ml}$ der zur Na- und K-Bestimmung verd. Grundlösung verwendeten wir unmittelbar für die Phosphat-Bestimmung nach FISKE-SubBAROW (20).

3. Calciumbestimmung: $5 \mathrm{ml}$ der salzsauren Ausgangslösung pipettierten wir in ein Zentrifugenglas, neutralisierten mit konz. $\mathrm{NH}_{4} \mathrm{OH}$, säuerten mit Essigsäure an und fällten das Calcium mit $2 \mathrm{~m} l$ gesätt. Ammoniumoxalatlösung bei $37^{\circ}$ aus. Nach $2-3$ stdg. Fällen zentrifugierten wir ab; dekantierten und wuschen mit $1 \% \mathrm{NH}_{4} \mathrm{OH}$ nach. Der Niederschlag wurde in wenigen Tropfen $2 n \mathrm{HCl}$ aufgenommen und mit $\mathrm{H}_{2} \mathrm{O}$ auf $100 \mathrm{ml}$ verdünnt. In dieser Lösung bestimmten wir das Calcium flammenphotometrisch. 4. Magnesiumbestimmung: Das Uberstehende und die Waschwässer von der Calciumoxalatfällung wurden mit $1,0 \mathrm{ml}$ gesätt. $\left(\mathrm{NH}_{4}\right)_{2}$
$\mathrm{HPO}_{4}$-Lsg. versetzt und mit Ammoniak auf $\mathrm{pH}=6,0$ cingestellt (Indikatorpapier Merck). Zur vollständigen Fällung des $\mathrm{MgNH}_{4} \mathrm{PO}_{4}$ wurde $12 \mathrm{Stdn}$. abgewartet. Dann setzten wir $1 \mathrm{ml}$ konz. $\mathrm{NH}_{4} \mathrm{OH} \mathrm{zu}$, ließen nochmals $3 \mathrm{Stdn}$. stehen und zentrifugierten das $\mathrm{MgNH}_{4} \mathrm{PO}_{4}$ ab. Den Niederschlag lösten wir, ohne zu waschen, in $1 n \mathrm{HCl}$ und füllten mit aq. dest. auf $25 \mathrm{ml}$ auf. Von dieser Lösung mischten wir $0,5 \mathrm{ml}$ mit $5,5 \mathrm{ml}$ aq. dest. und nahmen dann 5,0 $\mathrm{m} l$ zur Bestimmung des Magnesiums nach der Titangelbmethode (21). Die Messung des stabilisierten Magnesiumlackes erfolgte bei $540 \mathrm{~m} \mu$ in $5 \mathrm{~cm}$ Küvetten im Spektralphotometer gegen Reagenzienleerwert. Eine Eichkurve aus $\mathrm{MgNH}_{4} \mathrm{PO}_{4}$ lief mit.

Zur Oxalatbestimmung wurde $1 \mathrm{~g}$ Harntrockensubstanz mit $3 \mathrm{ml}$ $\mathrm{H}_{2} \mathrm{O}$ und $1 \mathrm{ml} 2 n \mathrm{HCl}$ versetzt, mehrmals umgeschüttelt und zentrifugiert. Der Uberstand und die 2 maligen salzsauren Waschwässer wurden nach Zusatz von $1,0 \mathrm{~m} / 15$ proz. $\mathrm{CaCl}_{2}$-Lsg. mit $2 n \mathrm{NaOH}$ alkalisiert und bei $37^{\circ} 2 \mathrm{Stdn}$. stehen gelassen. Nach abermaligem Zentrifugieren und Nachwaschen wurde das Sediment in $2 n$ Essigsäure gelöst. Der verbleibende Niederschlag (hauptsächlich Calciumoxalat) wurde einmal nachgewaschen und zur Abtrennung anhaftender reduzierender Substanzen noch einmal wie die Ausgangssubstanz behandelt bis die Stufe des in verd. Essigsäure unlöslichen Sedimentes abermals entstand. Dieses Sediment wurde dann nach Lösen in warmer verdünnter $\mathrm{H}_{2} \mathrm{SO}_{4}$ mit $0,01 n \mathrm{KMnO}_{4}$-Lösung titriert. War der Umschlagspunkt unscharf, wurde die Bestimmung verworfen und mit einer neuen Einwaage die Fällungsreihe zur besseren Reinigung des Calciumoxalates nicht $2 \mathrm{mal}$ sondern $3 \mathrm{mal}$ durchgeführt.

Harnkolloide: Ein anderer aliquoter Teil des Harnes wurde unter $\mathrm{N}_{2}$-Atmosphäre ultrafiltriert (Ultrafeinfilter lsg. 60 Gerät MD 140 der Membranfiltergesellschaft Göttingen). Den Rückstand der Harnkolloide nahmen wir quantitativ ab; trockneten ihn und bestimmten sein Gewicht. Um vergleichbare Angaben über die Harnkolloide machen zu können, ermittelten wir noch jeweils ihren Aschegehalt und berechneten dann als aschefreie HarnkolioidTrockensubstanz.

Die bakteriell entzündlicben Veränderungen des Harns bestimmten wir auf Grund der Leukozyten pro Gesichtsfeld und des Stäbchenund Kokkenbefundes im Methylenblaupräparat. Als normal betrachteten wir $0-5$ Leukozyten pro Gesichtsfeld und einen negativen Stäbchen- und Kokkenbefund. Höhere Leukozytenzahlen und Stäbchen oder Kokken deuteten wir als Zeichen einer Infektion.

\section{Ergebnisse}

Bei Gesunden (Gruppe a) schwankte die Tagesausscheidung von Calcium zwischen 61 und $251 \mathrm{mg}$, von Oxalat $z$ wischen 5 und $31 \mathrm{mg}$ und von anorgan. Phosphat (berechnet als P) zwischen 426 und $960 \mathrm{mg}$ (Tab. 1). Eine Harninfektion bestand nicht. Die Harnkolloidausscheidung lag $z$ wischen 81 und $305 \mathrm{mg} / \mathrm{Tag}$. Diese Werte entsprechen den aus der Literatur bekannten Normalwerten der täglichen Calcium-Oxalat- und Phosphatausscheidung im Harn. Um die Löslichkeit des Calciumoxalats im Harn beurteilen zu können, errechneten wir die molaren Konzentrationen der Ca- und Oxalationen und der Ionen der Lösungsvermittler im 24 Stdn.-Harn. Dazu wurde die in $1 \mathrm{~g}$ Trockensubstanz bestimmte Ionenmenge auf die Gesamtmenge der Trockensubstanz eines dem 24-Stdn.-Harn entnommenen bekannten Harnvolumens umgerechnet und die Molarität ermittelt. Das aus diesen Werten erhaltene Ionenprodukt setzten wir zur molaren Konzentration der Lösungsvermittler in Beziehung. Die Ionenprodukte und die erhaltenen Quotienten können dann beim Vergleich mit den Werten bei Steinkranken ein $\mathrm{MaB}$ für $\mathrm{Abweichungen} \mathrm{von} \mathrm{der}$ 
normalen Löslichkeit des Calciumoxalates im Harn sein. Das anorgan. Phosphat wurde mit in die Betrachtungen der Lösungsvermittler einbezogen, weil es im $\mathrm{HPO}_{4}{ }^{\prime \prime}$ oder $\mathrm{H}_{2} \mathrm{PO}_{4}{ }^{\prime}$ als zwei- oder einwertiger Fremdelektrolyt wirkt. Die Ionenprodukte des Calciumoxalats im Harn der Gesunden (Tab. 1) liegen alle mit $0,14-1,46 \times 10^{-6}$ über dem Löslichkeitsprodukt des Calciumoxalats von 3-7 $\times 10^{-9}$. Ohne Lösungsvermittler würde also Normalharn an Calciumoxalat übersättigt und kristallisationsbereit sein. Um die Löslichkeit von Calciumoxalat im Normalharn trotzdem aufrecht $\mathrm{zu}$ erhalten sind pro Ionenprodukt des Calciumoxalats von $10^{-6}$ mindestens $3,2 \times 10^{-3} \mathrm{Mol} / l$ Magnesium, $19 \times 10^{-3} \mathrm{Mol} / l$ anorg. Phosphat (berechnet als P) und $106 \times 10^{-3} \mathrm{Mol} / l \mathrm{Na}$ trium und Kalium vorhanden. Wir berechneten außerdem das $[\mathrm{Ca}] \times\left[\mathrm{P}_{\text {anorg }}\right]$-Produkt, obwohl es für die Unlöslichkeit von Calciumphosphat im Normalharn keine Bedeutung hat, weil die notwendigen $\mathrm{pH}$-Werte von 6,5 nicht oder nur kurzfristig überschritten werden. Es beträgt $38-130 \times 10^{-6}$ (Tabelle 1 ). Wir wollten damit Richtwerte gewinnen, mit denen später die [Ca] $\times[\mathrm{P}]$-Produkte infizierter Harne verglichen werden können. Aus dem gleichen Grunde bestimmten wir, wieviel Lösungsvermittler pro $[\mathrm{Ca}] \times[\mathrm{P}]$-Produkt von $10^{-6}$ im Liter Normalharn vorkommen. Für Magnesium fanden wir $2,2 \times 10^{-5} \mathrm{Mol} / l$ und für Natrium und Kalium $0,8 \times 10^{-3} \mathrm{Mol} / l$ als Mindestwert.

Unter 15 Kranken mit Calciumoxalatsteinen (Gruppe b) fanden wir nur einmal eine erhöhte Calcium-Tagesausscheidung und zweimal eine erhöhte Oxalat-Tagesausscheidung (Tab. 2). Durch Infektion bedingte $\mathrm{pH}-\mathrm{Verschiebungen} \mathrm{des} \mathrm{Harnes} \mathrm{haben} \mathrm{auf} \mathrm{die} \mathrm{Lös-}$ lichkeit von Calciumoxalat keinen Einfluß und scheiden deshalb bei diesen Fällen aus der Betrachtung aus. Ordnet man nun, um ein $\mathrm{Ma} \beta$ für die wirklichen Löslichkeitsverhältnisse des Calciumoxalats zu bekommen, die Meßwerte nach der Höhe der Lösungsvermittler-Konzentration pro Ionenprodukt des Calciumoxalats von $10^{-6}$, so ergibt sich ein anderes Bild (Tab. 3). Alle in der Tabelle 3 fettgedruckten Werte veranschaulichen schlechtere Löslichkeitsbedingungen für Calciumoxalat im Vergleich zu gesunden Personen. Bei 5 Patienten mit Calciưmoxalatsteinen ist die Magnesiumkonzentration im Verhältnis zum aktuellen Ionenprodukt des Calciumoxalats im Harn

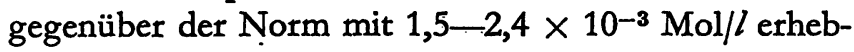
lich verringert. Bei 4 dieser Patienten ist auch die anorg. Phosphatkonzentration mit 10,8-16,4 Mol $/ /$ pro [Ca] $[\mathrm{Ox}]=10^{=6}$ herabgesetzt. Bei zwei dieser Patienten werden gleichzeitig nur $6,3 \times 10^{-3} \mathrm{Mol} / l[\mathrm{Na}]$ und $[\mathrm{K}]$ pro $[\mathrm{Ca}][\mathrm{Ox}]=10^{-6}$ ausgeschieden. Das Ionenprodukt des Calciumoxalats [Ca] [Ox] im Harn selbst ist nur bei 2 der genannten 5 Patienten im Vergleich zu Gesunclen erhöht. Die Konzentration der ausgeschiedenen Harnkolloide unterscheidet sich kaum von der bei Gesunden. Man findet bei Steinkranken mit 23,8-51 mg\% eher einzelne erhöhte Harnkolloidkonzentrationen. Patient Wu. mit einem gemischten Calciumoxalat/Harnsäurestein hatte mit $6,6 \times 10^{-3} \mathrm{Mol} / l$ als einziger der Steinkranken eine stark erhöhte Harnsäurekonzentration im Harn (normal 2,4 $\times 10^{-3} \mathrm{Mol} / \mathrm{l}$ ).

Bei Patienten mit calciumbaltigen, Rö-Schatten gebenden Steinen, deren Steinzusammensetzung wir nicht bestimmen konnten, fanden wir sehr viel häufiger über die Norm erhöhte Calcium- und Oxalat-Tagesausscheidungen (Tab. 4). Von 16 Patienten schieden $3 \mathrm{mehr}$ Calcium, 5 mehr Oxalat und $1 \mathrm{mehr}$ Calcium und Oxalat als Gesunde täglich aus. Die Tagesausscheidung an anorg. Phosphor unterschied sich nicht von der bei Gesunden. Bei diesen Patienten untersuchten wir die Löslichkeitsbedingungen für Calciumoxalat und Calciumphosphat getrennt. Zunächst gingen wir davon aus, da $B$ alle Patienten Calciumoxalatsteine hätten (Tab. 5). Die fettgedruckten Werte zeigen wieder die schlechteren Löslichkeitsbedingungen für Calciumoxalat im Vergleich zu Gesunden. Dabei ergab sich, da 7 von 16 Patienten nur 5,0-15,9 $\times 10^{-3} \mathrm{Mol} / l$ anorg. Phosphor pro $[\mathrm{Ca}][\mathrm{Ox}]=10^{-6}$ ausschieden. 6 dieser $\mathrm{Pa}$ tienten schieden gleichzeitig weniger Magnesium $\left(1,0-2,55 \times 10^{-3} \mathrm{Mol} / \mathrm{l}\right)$ und weniger Natrium $+\mathrm{Ka}-$ lium $\left(34,4-88,3 \times 10^{-3} \mathrm{Mol} / 2\right)$ pro [Ca] [Ox] $=10^{-6}$ als Gesunde aus. 5 dieser Patienten hatten ein erhöhtes [Ca] [Ox]-Ionenprodukt $\left(1,58-4,68 \times 10^{-6}\right)$ im Harn. Die Konzentration der Harnkolloide war bei allen $\mathrm{Pa}$ tienten dieser Gruppe unauffällig. Die Löslichkeitsbedingungen für Calciumphosphat untersuchten wir bei den Steinpatienten mit infizierten Harnen, weil bei ihnen durch neutralen oder alkalischen Harn die Voraussetzungen einer hinreichenden Herabsetzung der Löslichkeit der Calciumphosphate gegeben sein und das aktuelle Ionenprodukt von Calcium und Phosphat das Löslichkeitsprodukt eines Calciumphosphates überschreiten kann (Tab. 6). Unter 9 Fällen lag nur einmal die Magnesiumkonzentration pro $[\mathrm{Ca}][\mathrm{P}]=10^{-6}$ mit $1,7 \times 10^{-5}$ $\mathrm{Mol} / l$ unterhalb der Norm. Das [Ca] [P]-Produkt und die $[\mathrm{Na}]+[\mathrm{K}]$-Konzentrationen pro $[\mathrm{Ca}][\mathrm{P}]=10^{-6}$ schwankten innerhalb der Werte bei Gesunden. Die Harninfektion blieb also der einzige Faktor bei diesen 9 Patienten, der die Calciumphosphatlöslichkeit durch $\mathrm{pH}-$ Änderung herabsetzen konnte.

\section{Diskussion}

Unsere Ergebnisse ergänzen die Untersuchungen von HAMmarsten (4) über die Löslichkeit des Calciumoxalats im Rattenharn. Hammarsten (4) beobachtete, $\mathrm{da} ß$ Calciumoxalat im Rattenharn bei einem Calciumoxalationenprodukt von $1,14 \times 10^{-6}$ und einer $\mathrm{Ma}$ gnesiumkonzentration von $15-31 \times 10^{-3} \mathrm{Mol} / l$ (das entspricht pro $10^{-6}[\mathrm{Ca}$ ] [Ox] einer Magnesiumkonzentration von $\left.13-28 \times 10^{-3} \mathrm{Mol} / \mathrm{l}\right)$ stets vollständig in Lösung gehalten wurde. Wir sehen, daß im Harn gesunder Menschen Calciumoxalat bei einem Calciumoxalationenproduktvon 0,14-1,46 $\times 10^{-6}$ und einer Magnesiumkonzentration von $3,2-25,7 \times 10^{-3} \mathrm{Mol} / l$ pro $10^{-6}$ [Ca] [Ox] in Lösung gehalten wird. Im Rattenharn bestand nach HaMmARSTEN bei einem [Ca] [Ox] von $3,18 \times 10^{-6}$ und einer Magnesiumkonzentration von $6 \times 10^{-3} \mathrm{Mol} / /$ (das entspricht pro $10^{-6}[\mathrm{Ca}][\mathrm{Ox}]$ einer Magnesiumkonzen- 
Tab. 1

Untersuchungen des 24-Stdn.-Harnes von Gesunden*)

\begin{tabular}{|c|c|c|c|c|c|c|c|c|c|c|c|c|c|}
\hline & $\begin{array}{l}\text { ml } \\
\text { Harn } \\
\text { tgl. }\end{array}$ & $\begin{array}{l}\mathrm{mg} \\
\mathrm{Ca} \\
\mathrm{tgl} .\end{array}$ & $\begin{array}{l}\mathrm{mg} \\
\text { Oxalat } \\
\text { tgl. }\end{array}$ & $\begin{array}{c}\mathrm{mg} \\
\mathrm{t} \text { anorg. } \mathrm{P} \\
\text { tgl. }\end{array}$ & $\begin{array}{c}\text { mg } \\
\text { aschefreie } \\
\text { Harn- } \\
\text { kolloid } \\
\text { T. S./tgl. }\end{array}$ & $\begin{array}{l}{[\mathrm{Ca}[\times[\mathrm{Ox}]} \\
\text { Ionen- } \\
\text { produkt }\end{array}$ & $\underset{ }{[\mathrm{Mg}]}$ & $\begin{array}{l}\text { [P anorg] } \\
\text { a] [Ox]-Ionen } \\
\text { von 10-6 }\end{array}$ & $\begin{array}{l}{[\mathrm{Na}]+[\mathrm{K}]} \\
\text { nprodukt }\end{array}$ & $\begin{array}{c}\text { mg } \% \\
\text { aschefreie } \\
\text { Harn- } \\
\text { kolloid } \\
\text { T. S./tgl. }\end{array}$ & $\begin{array}{c}{[\mathrm{Ca}] \times[\mathrm{P}]} \\
\text { produkt }\end{array}$ & $\begin{array}{l}{[\mathrm{Mg}]} \\
\text { pro [Ca] } \\
\text { produkt }\end{array}$ & $\begin{array}{l}{[\mathrm{Na}]+[\mathrm{K}]} \\
\mid[\mathrm{P}]-\text { Ionen- } \\
t \text { von } 10^{-6}\end{array}$ \\
\hline Du ô & 1130 & 217 & 31 & 960 & 305 & $1,46 \times$ & $3,2 \times 10^{-3}$ & $19 \times 10^{-3}$ & $3106 x$ & 19,7 & 130 & $3,7 \times 10^{-5}$ & 1,2 \\
\hline Ta 9 & 825 & 61 & 28 & 578 & 96 & $0,74 \times 10^{-6}$ & $8,7 \times 10^{-3}$ & $30,5 \times 10^{-3}$ & $3344 \times 10^{-3}$ & 10,8 & $43 \times 10^{-6}$ & $15,9 \times 10^{-5}$ & $5,9 \times 10^{-3}$ \\
\hline $\mathrm{Ja} \quad \delta$ & 1410 & 251 & 14 & 797 & 103 & $0,50 \times 10^{-6}$ & $6,8 \times 10^{-3}$ & $36,4 \times 10^{-3}$ & $3490 \times 10^{-3}$ & 8,1 & $82 \times 10^{-6}$ & $4,1 \times 10^{-5}$ & $3,0 \times 10^{-3}$ \\
\hline $\mathrm{Ma} \sigma^{\pi}$ & 610 & 130 & 7 & 426 & 87 & $0,69 \times 10^{-6}$ & $4,8 \times 10^{-3}$ & $32,8 \times 10^{-3}$ & $3391 \times 10^{-3}$ & 8,4 & $120 \times 10^{-6}$ & $2,8 \times 10^{-5}$ & $2,3 \times 10^{-3}$ \\
\hline $\mathrm{Pe}$ क & 990 & 93 & 5 & 504 & 84 & $0,14 \times 10^{-6} 2$ & $25,7 \times 10^{-3}$ & $117,5 \times 10^{-3}$ & $3930 \times 10^{-3}$ & 6,6 & $38 \times 10^{-6}$ & $9,5 \times 10^{-5}$ & $3,4 \times 10^{-3}$ \\
\hline Bü $\tilde{\sigma}$ & 710 & 134 & 9 & 494 & 175 & $0,71 \times 10^{-6}$ & $3,2 \times 10^{-3}$ & $31,8 \times 10^{-3}$ & $3317 \times 10^{-3}$ & 10,9 & $106 \times 10^{-6}$ & $2,2 \times 10^{-5}$ & $2,1 \times 10^{-3}$ \\
\hline $\mathrm{Br}$ 우 & 860 & 118 & 7 & 667 & 99 & $0,31 \times 10^{-6}$ & $8,7 \times 10^{-3}$ & $80,5 \times 10^{-3}$ & $3226 \times 10^{-3}$ & 9,0 & $85 \times 10^{-6}$ & $3,2 \times 10^{-5}$ & $0,8 \times 10^{-3}$ \\
\hline
\end{tabular}

*) Nicht infizierte Harne

[] bedeutet $\mathrm{Mol} / \mathrm{l}$

Tab. 2

Tagesausscheidung vom Calium, Oxalat und Harnkolloid bei Patienten mit Calciumoxalatsteinen

Name Infektion $\quad \mathrm{m} / \mathrm{Harn} \quad \mathrm{mgCa} \quad \mathrm{mg}$ Oxalat \begin{tabular}{c}
$\begin{array}{c}\mathrm{mg} \\
\text { aschefreie } \\
\text { Harn- } \\
\text { kolloid } \\
\text { T.S. }\end{array}$ \\
\hline
\end{tabular}

Über $252 \mathrm{mg}$ Calcium tgl.

\begin{tabular}{|c|c|c|c|c|c|c|}
\hline $\mathrm{He}$ & $\hat{\sigma}$ & $\varnothing$ & 1390 & 304 & 14 & 186 \\
\hline \multicolumn{7}{|c|}{ Úber 31 mg Oxalat tgl. } \\
\hline $\mathrm{Me}$ & $\hat{\sigma}$. & + & 1850 & 230 & 55 & - \\
\hline Wu & 우 & + & 635 & 81 & 50 & - \\
\hline \multicolumn{7}{|c|}{$\mathrm{Ca}_{\mathrm{a}}$ und Oxalatausscheidung normal } \\
\hline $\mathrm{Kl}$ & q & + & 1166 & 91 & 20 & 548 \\
\hline $\mathrm{Ha}$ & ot & $\varnothing$ & 800 & 155 & 7 & 356 \\
\hline We & 오 & + & 400 & 59 & 2 & - \\
\hline Mei & o & $\varnothing$ & 1265 & 145 & 9 & - \\
\hline $\mathrm{Hau}$ & $\sigma$ & $\widetilde{\varnothing}$ & 740 & 47 & 2 & 159 \\
\hline $\mathrm{Bl}$ & 우 & + & 965 & 119 & 12 & - \\
\hline Sch & $0^{\pi}$ & $\varnothing$ & 1140 & 171 & 19 & 119 \\
\hline $\mathrm{Be}$ & 오 & $\varnothing$ & 300 & 78 & 5 & 325 \\
\hline Go & $\sigma^{\pi}$ & $\varnothing$ & 655 & 61 & 13 & 24 \\
\hline $\mathrm{Li}$ & 우 & + & 350 & 158 & 5 & 158 \\
\hline Scho & q & + & 430 & 37 & 7 & - \\
\hline re & 우 & + & 1230 & 188 & 27 & - \\
\hline
\end{tabular}

tration von $\left.1,89 \times 10^{-3} \mathrm{Mol} / l\right)$, die Gefahr einer Calciumoxalatkristallisation. Bei einem [Ca] [Ox]-Produkt von $44 \times 10^{-6}$ und einer Magnesiumkonzentration von $6 \times 10^{-3} \mathrm{Mol} / l$ entsprechend einer Magnesiumkonzentration von $0,14 \times 10^{-3} \mathrm{Mol} / l$ pro $10^{-6}$ [Ca] [Ox] fand sich bereits in etwa $60 \%$ der Fälle massive Calciumoxalatsteinbildung.

7 unserer 31 untersuchten Steinkranken hatten im Harn ein [Ca] [Ox]-Produkt zwischen 1,58-4,68 $\times 10^{-6}$, 11 Patienten eine Magnesiumkonzentration von 1,0 $2,55 \times 10^{-3} \mathrm{Mol} / l$ pro $10^{-6}$ [Ca] [Ox]. Die Löslichkeitsbedingungen für Calciumoxalat in Gegenwart von Magnesium weichen demnach im Harn des Menschen nicht . wesentlich von denen im Rattenharn ab. Wenn das Verhältnis zwischen Magnesium-Konzentration und
Tab. 3

Ionenprodukt des Calciumoxalats und Konzentration der kristalloiden Lösungsvermittler pro $[\mathrm{Ca}] \times[\mathrm{Ox}]=10^{-6}$ im Harn von Patienten mit Calciumoxalatsteinen

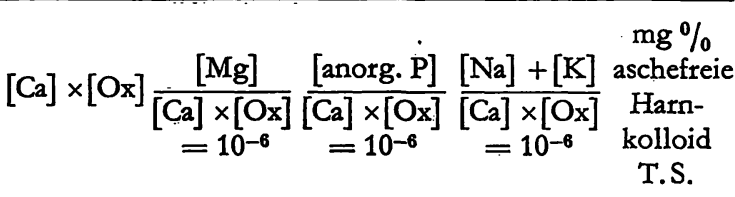

Von der Norm abweichende Werte

Wu $\quad 2,88 \times 10^{-6} \quad 2,2 \times 10^{-3} \quad 16,4 \times 10^{-3} \quad 63 \times 10^{-3}$

$\mathrm{Li}$ i $1,81 \times 10^{-6} \quad 1,7 \times 10^{-3} \quad 14,2 \times 10^{=3} \quad 128 \times 10^{-3} \quad 18,7$

Me ơ $1,08 \times 10^{-6} \quad 1,6 \times 10^{-3} \quad 10,8 \times 10^{-3} \quad 64 \times 10^{-3} \quad-$

$\operatorname{Re}$ \& $0,96 \times 10^{-6} \quad 1,5 \times 10^{-3} \quad 11,3 \times 10^{-3} \quad 112 \times 10^{-3} \quad-$

$\mathrm{Gu}$ o $0,53 \times 10^{-6} \quad 2,4 \times 10^{-3} \quad 24,3 \times 10^{-3} \quad 191 \times 10^{-3} \quad 2,6$

\section{Normalwerte}

$\mathrm{Be}$ \& $1,24 \times 10^{-6} \quad 4,4 \times 10^{-3} \quad 17,1 \times 10^{-3} \quad 869 \times 10^{-3} \quad 51,0$

$\mathrm{Kl}$ \& $0,39 \times 10^{-6} \quad 5,6 \times 10^{-3} \quad 37,8 \times 10^{-3} \quad 161 \times 10^{-3} \quad 23,8$

$\mathrm{Ha}$ o $0,49 \times 10^{-6} \quad 12,8 \times 10^{-3} \quad 45,2 \times 10^{-3} \quad 197 \times 10^{-3} \quad 23,8$

We . O $0,22 \times 10^{-6} 19,9 \times 10^{-3} 101,0 \times 10^{-3} 392 \times 10^{-3}$

Mei \& $0,35 \times 10^{-6} \quad 8,0 \times 10^{-3} \quad 59,0 \times 10^{-3} 311 \times 10^{-3} \quad-$

Hau $\sigma^{\top} 0,05 \times 10^{-6} 28,0 \times 10^{-3} 176,0 \times 10^{-3} 239 \times 10^{-3} \quad 8,3$

$\mathrm{He}$ o $0,60 \times 10^{-6} \quad 5,2 \times 10^{-3} \quad 29,1 \times 10^{-3} \quad 242 \times 10^{-3} \quad 9,0$

Bl o $0,43 \times 10^{-6} \quad 6,3 \times 10^{-3} \quad 41,5 \times 10^{-3} \quad 505 \times 10^{-3} \quad-$

Sch ot $0,71 \times 10^{-6} \quad 4,8 \times 10^{-3} \quad 21,4 \times 10^{-3} \quad 203 \times 10^{-3} \quad 9,3$

Scho $0,41 \times 10^{-6} 26,3 \times 10^{-3} \quad 63,2 \times 10^{-3} \quad 354 \times 10^{-3}$

[Ca] [Ox]-Produkt zu ungünstig wird, treten anscheinend auch beim Menschen Harnsteine auf. Parallel zum Magnesium ist bei den meisten der 11 Steinkranken auch die Konzentration des anorg. Phosphats und von $[\mathrm{Na}]+[\mathrm{K}]$ pro $10^{-6}[\mathrm{Ca}][\mathrm{Ox}]$ abgesunken. Diese Lösungsvermittler ergänzen in vivo, wie nach Berechnungen (3) und in vitro Versuchen $(4,5) \mathrm{zu}$ erwarten ist, die Wirkung des Magnesiums auf die Löslichkeit des Calciumoxalats. Unsere Untersuchungen bestätigen schließlich unsere schon früher vertretene Auffassung $(1,5)$, daß bei Steinkranken die Konzentration der Harnkolloide nicht verringert ist und diese deshalb als kolloidale Lösungsvermittler für Calciumsalze ausscheiden. Bei Steinkranken mit schattengebenden Konkrementen, deren Struktur wir durch Steinanalyse nicht 
Tab. 4

Tagesausscheidung von Calcium, Oxalat, anorg. Phosphor und Harnkolloid

bei Patienten mit Rö-Scbatten gebenden Steinen

\begin{tabular}{cccccc}
\hline Name Infektion & & & & aschefreie \\
Harn & Calcium \\
$\mathrm{mg}$ & $\mathrm{mg}$ & $\mathrm{mg}$ & $\mathrm{mg}$ & $\mathrm{mg}$ T.S. \\
\hline
\end{tabular}

Uber $252 \mathrm{mg} \mathrm{Ca} \mathrm{tgl.}$

\begin{tabular}{|c|c|c|c|c|c|c|}
\hline Mü o & $\varnothing$ & 1354 & 288 & 23 & 678 & 603 \\
\hline Heu 9 & $\varnothing$ & 820 & 337 & 10 & 493 & - \\
\hline Bö & + & 1860 & 446 & .20 & 1050 & \\
\hline
\end{tabular}

Über $31 \mathrm{mg}$ Oxalat tgl.

\begin{tabular}{|c|c|c|c|c|c|c|}
\hline $\mathrm{Ba}$ 우 & & 1220 & 216 & 45 & 702 & - \\
\hline Web ${ }^{*}$ & + & 983 & 165 & 40 & 670 & - \\
\hline $\mathrm{Ju} \quad \bar{\sigma}$ & + & 1540 & 53 & 60 & 540 & - \\
\hline Ho 0 & + & 1460 & 100 & 67 & 610 & 130 \\
\hline Mar & + & 740 & 163 & 55 & 540 & 137 \\
\hline
\end{tabular}

Uber $252 \mathrm{mg} \mathrm{Ca}$ tgl. und über $31 \mathrm{mg}$ Oxalat tgl.

\begin{tabular}{|c|c|c|c|c|c|c|}
\hline $\mathrm{Lu} \sigma^{\pi}$ & + & 2525 & 388 & 91 & 1200 & 179 \\
\hline \multicolumn{7}{|c|}{$\mathrm{Ca}$-Oxalatausscheidung normal } \\
\hline Qu $\quad \sigma^{\pi}$ & $\varnothing$ & 1050 & 183 & 23 & 998 & 144 \\
\hline Har $\sigma^{\pi}$ & + & 1700 & 188 & 8 & 617 & - \\
\hline $\mathrm{Za} \sigma^{\pi}$ & + & 850 & 100 & 24. & 658 & - \\
\hline 우 & + & 1140 & 171 & 5 & 863 & 104 \\
\hline $\mathrm{Mi} \sigma^{\pi}$ & $\varnothing$ & 540 & 64 & 34 & 487 & - \\
\hline Jung $\sigma^{*}$ & $\tilde{\varnothing}$ & 1420 & 218 & 18 & 860 & - \\
\hline Schuo & + & 1270 & 74 & 17 & 395 & 259 \\
\hline
\end{tabular}

Tab. 5

Ionenprodukt des $[\mathrm{Ca}] \times[\mathrm{Ox}]$ und Konzentration der kristalloiden Lösungsvermittler pro $[\mathrm{Ca}] \times[\mathrm{Ox}]=10^{-6}$ im Harn von Patienten mit Rö-Scbatten gebenden Steinen

$\mathrm{mg} \%$

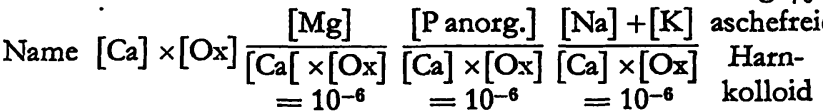
T.S.

Von der Norm abweichende Werte

Web ô $1,94 \times 10^{-6} 2,55 \times 10^{-3} \quad 11,3 \times 10^{-3} \quad 80 \times 10^{-3}$ $\mathrm{Lu}$ ơ $1,58 \times 10^{-6} 1,0 \times 10^{-3} \quad 9,7 \times 10^{-3} \quad 55,4 \times 10^{-3}$ $\mathrm{Ba}$ ㅇ $1,86 \times 10^{-6} \quad 1,71 \times 10^{-3} \quad 10,0 \times 10^{-3} \quad 64,2 \times 10^{-3}$ Mat o $4,68 \times 10^{-6} 1,12 \times 10^{-3} \quad 5,0 \times 10^{-3} \quad 34,4 \times 10^{-3}$ $\mathrm{Mi}$ o $2,12 \times 10^{-6} 1,05 \times 10^{-3} \quad 13,7 \times 10^{-3} \quad 87,0 \times 10^{-3}$ Mü \& $1,01 \times 10^{-6} 3,41 \times 10^{-3} \quad 15,9 \times 10^{-3} \quad 88,3 \times 10^{-6}$ Ho of $0,89 \times 10^{-6} 2,42 \times 10^{-3} \quad 15,2 \times 10^{-3} 165,0 \times 10^{-3}$

Werte im Bereich der Norm

Qu $\sigma^{\times} 1,09 \times 10^{-6} 3,4 \times 10^{-3} \quad 28,0 \times 10^{-3} 147,0 \times 10^{-3}$ Har o $0,14 \times 10^{-6} 13,5 \times 10^{-3} \quad 83,5 \times 10^{-3} 835 \times 10^{-3}$ $\mathrm{Ju} \quad 0,37 \times 10^{-6} 9,2 \times 10^{-3} 30,3 \times 10^{-3} 196 \times 10^{-8}$

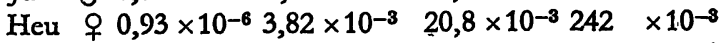
Bö $\delta^{-1} 0,72 \times 10^{-6} 5,3 \times 10^{-3} \quad 25,3 \times 10^{-3} 196,5 \times 10^{-3}$ $\mathrm{Za}$ व $0,94 \times 10^{-6} 3,5 \times 10^{-3} \quad 26,5 \times 10^{-3} 187 \times 10^{-3}$ $\mathrm{Bi} \quad \mathrm{O} 0,19 \times 10^{-6} 8,25 \times 10^{-3} 128,5 \times 10^{-3} 870 \times 10^{-3} \quad 11,0$ Jung ot $0,54 \times 10^{-6} 9,8 \times 10^{-3} 36,1 \times 10^{-8} 251 \times 10^{-3}-$ Schu 우 $0,22 \times 10^{-6} 8,2 \times 10^{-8} \quad 45,5 \times 10^{-3} 375 \times 10^{-3} \quad 16,7$

[] bedeutet Mol/l
Tab. 6

[Ca] [P]-Produkt und Konzentration der kristalloiden Lösungsvermittler pro $[\mathrm{Ca}][\mathrm{P}]=10^{-6}$

in infizierten Harnen von Patienten mit Rö-dichten Steinen

\begin{tabular}{|c|c|c|c|}
\hline \multirow{2}{*}{ Name } & \multirow{2}{*}[\mathrm{Ca}]{$[\mathrm{P}]$} & {$[\mathrm{Mg}]$} & {$[\mathrm{Na}]+[\mathrm{K}]$} \\
\hline & & {$[\mathrm{Ca}][\mathrm{P}]=10^{-6}$} & {$[\mathrm{Ca}][\mathrm{P}]=10^{-6}$} \\
\hline $\mathrm{Bi}$ ㅇ & $92 \times 10^{-6}$ & $1,7 \times 10^{-5}$ & $1,8 \times 10^{-6}$ \\
\hline Bö ठే & $109 \times 10^{-6}$ & $3,5 \times 10^{-5}$ & $1,3 \times 10^{-6}$ \\
\hline We ơ & $92 \times 10^{-6}$ & $5,4 \times 10^{-5}$ & $1,69 \times 10^{-6}$ \\
\hline Har $\sigma^{\pi}$ & $32 \times 10^{-6}$ & $5,9 \times 10^{-5}$ & $3,67 \times 10^{-6}$ \\
\hline Ju $\sigma^{*}$ & $10 \times 10^{-6}$ & $34,0 \times 10^{-5}$ & $7,26 \times 10^{-6}$ \\
\hline $\mathrm{Za} \quad \sigma^{\pi}$ & $74 \times 10^{-6}$ & $4,4 \times 10^{-5}$ & $2,38 \times 10^{-6}$ \\
\hline Ho $\sigma^{\pi}$ & $23 \times 10^{-6}$ & $9,4 \times 10^{-5}$ & $6,35 \times 10^{-6}$ \\
\hline Ma $\tilde{\delta}$ & $130 \times 10^{-6}$ & $4,0 \times 10^{-5}$ & $1,24 \times 10^{-6}$ \\
\hline $\mathrm{Lu} \sigma^{\pi}$ & $59 \times 10^{-6}$ & $2,7 \times 10^{-5}$ & $1,49 \times 10^{-6}$ \\
\hline
\end{tabular}

[ ] bedeutet $\mathrm{Mol} / \mathrm{l}$

ermitteln konnten, scheint die Unlöslichkeit von Calciumphosphaten nur durch einen infektionsbedingten $\mathrm{pH}$ Anstieg. des Harnes möglich zu sein. Abgesehen von einem Fall waren bei allen übrigen Patienten der Gruppe (c) mit infizierten Harnen die Konzentrationen der Lösungsvermittler unverändert.

Man wird nun fragen, warum nicht alle Steinkranken Veränderungen der Konzentrationen der Lösungsvermittler gegenüber den Ionenprodukten von [Ca] [Ox] oder [Ca] [P] im Harn aufweisen. Von 15 Kranken mit reinen Oxalatsteinen können wir nur 5 Fälle durch verschlechterte Löslichkeitsbedingungen für Calciumoxalat erklären. Von 16 Kranken mit Rö-Schatten gebenden Steinen können wir 3 Fälle durch verschlechterte Löslichkeitsbedingungen für Calciumoxalat, 4 durch verschlechterte Löslichkeitsbedingungen für Calciumoxalat und Calciumphosphat und 6 weitere Fälle durch verschlechterte Löslichkeitsbedingungen für Calciumphosphat erklären. Nur bei 3 Patienten der Gruppe fanden wir gegenüber Gesunden weder für Calciumoxalat noch für Calciumphosphat schlechtere Löslichkeitşbedingungen. Insgesamt lassen sich also von 31 Harnsteinbildungen nach unseren Untersuchungen 13 nicht durch Löslichkeitsveränderungen der Calciumsalze im Harn erklären. Der Grund hierfür ist z. T. in unserer Versuchsanordnung zu suchen. Mehrmalige Bestimmungen der Löslichkeit der Calciumsalze im Harn bei 8 Patienten in Abständen von einigen Tagen bis Wochen ergaben, daß die Löslichkeitsbedingungen der Calciumsalze nur bei einem Teil der Fälle (3) gleichmäßig schlecht blieben. Bei dem anderen Teil der Patienten war die schlechte Löslichkeit von Calciumoxalat im Harn nicht regelmäßig wiederzufinden. Das Harnsteinwachstum vollzieht sich wahrscheinlich auch nicht kontinuierlich, sondern in Perioden. Das könnte bedeuten, daß wir einen Teil unserer Steinkranken nicht in der Phase des Steinwachstums und der Kristallisationsbereitschaft der Calciumsalze im Harn exfaßt haben.

Unsere Untersuchungen zeigen, daß bei etwa der Hälfte der Steinkranken die Kristallisationsbereitschaft des 
Harnes als Voraussetzung der Steinbildung anhand verschlechterter Löslichkeitsbedingungen steinbildender Calciumsalze in Tagesproben tatsächlich nachzuweisen ist. Die hypothetische, aktiv ionenreichernde organische Matrix braucht man bei diesen Fällen zur Erklärung des Steinwachstums nicht. Der Matrix kommt eine Funktion als Kristallisationskern zu. Auch jeder andere Kristallisationskern im Harn könnte - unterstützt von langsamer Harnströmung - bei diesen Patienten genügen, um die Steinbildung auszulösen. Man sollte deshalb Untersuchungen der Konzentration kristalloider Lösungsvermittler im Harn und ihrer Beziehungen zu den aktuellen Ionenprodukten der Calciumsalze häufiger zur Diagnostik, Prognostik und zum Erkennen der Pathogenese des Harnsteinleidens heranziehen. Ursache der Konzentrationsänderungen kristalloider Lösungsvermittler und der Calciumsalze im Harn können tubuläre Schäden oder allgemeine Stoffwechselerkrankungen sein. Gerade die Calcium- und Magnesiumkonzentration des Harnes wird wesentlich durch tubuläre Funktionen mitbestimmt $(22,23)$. Beziehungen zwischen der Harninfektion und der Menge der ausgeschiedenen kristalloiden Lösungsvermittler oder der Menge der Harnkolloide lassen sich aus unseren Beobachtungen nicht ableiten.

\section{Literatur}

1. Dulce, H. J., Internist, 3, 684 (1962). - 2. Palocz, I. und I. Sugar, Acta med. Acad. Sci. hung. 3, 285 (1952). - 3. Meyer, J., Zschr. klin. Med., 111, 613 (1929). - 4. Hammarsten, G., Lunds Univ. Årskr. 32, Nr. 12 (1937). - 5. DuLCE, H. J., Hoppe-Seyler's Z. physiol. Chem. 311, 185 (1958), Hoppe-Seyler's Ż. physiol. Chem. 311, 191 (1958), Hoppe-Seyler's Z. physiol. Chem. 311, 197 (1958). - 6. Thomas, W. C. und J. E. Howard, Transact. Ass. Amer. Physicians 72, 181 (1959). - 7. Taupitz, E. und R. Schwerssfurth, Klin. Wschr. 39, 1256 (1961). - 8. Dulce, H. J., Unveröffentlichte Untersuchungen. - 9. Lrrin, R. B., G. R. Diessner und F. R. Keating, J. Urol. Baltimore 86,17 (1961). - 10. Cotret, J., Bull. Acad. nat. Méd. 144, 128 (1960). - 11. Cotret, J. und C. Virtu, Bull. med. 63, 878 (1955). -

12. Boyce, W. H., F. K. Garvey und C. E. Goven, J. Amer.
Med. Ass. 166, 1577 (1958). - 13. Dempsey, E. F., J. Clin. Invest.. 36, 882 (1957). - 14. HzRkeL, W. und K. Koch, Dtsch. Arch, klin. Med. 178, 511 (1936). - 15. Sutherdand, J. W., Brit. J. Urol. 26, 22 (1954). - 16. v. Sengbusch, R. und A. TimmermanN, Urol. Int. 4, 76 (1957). - 17. DuLcE, H. J., Aerztl. Wschr., 11, 445 (1956). - 18. KlemperkR, G. und F. Fritschler, Zschr. klin. Med., 44, 337 (1902). - 19. Krssin, B. und M. Locks, Proc. Soc. exp. Biol. Med. 46, 216 (1941). - 20. TAuprtz, E. und A. GACA, Urol. Int. 14, 15 (1962). - 21. Frske, C. H. und Y. Subbarow, J. biol. Chemistry 66, 375 (1925). - 22. Orange, $M$. und H. C. Rrein, J. biol. Chemistry 189, 379 (1951). - 23. Brull, I. und J. Berntrolin, Atch. int. Pharmacodynam. Thérap. 108, 330 (1956). - 24. Meriz, D. P., Klin. Wschr. 35, 1171 (1957).

Priv.-Doz. Dr. med. Hans-Joachim Dulce Physiologisch-chemisches Institut der Freien Universität Berlin 1 Berlin 33, Dahlem, Lentzeallee 75

\section{Buchbesprechung}

Dünnschicht-Chromatographie, Randerath, Kurt: (Monographie Nr. 78 zu Angewandte Chemie und Chemie-Ingenieur-Technik), XII, 243 S., 78 Abb., 77 Tab., 2 Farbtafeln. Verlag Chemie GmbH, Weinheim/Bergstr., 1962. DM 22,-. Die Dünnschicht-Chromatographie hat sich in erstaunlich kurzer Zeit im biochemischen, im klinisch-chemischen, im pharmazeutischen und pharmakologischen Laboratorium als analytische Methode durchgesetzt. Ein gutes Übersichtswerk über die Möglichkeiten, aber auch über die Begrenzung der Dünnschicht-Chromatographie kommt somit einem Bedürfnis entgegen. Das Buch von Randerath ist eine logische Ergänzung des hervorragenden Buches von Cramer aus dem gleichen Verlag über die Papier-Chromatographie. Der Autor gibt einleitend in einem kurzen allgemeinen Teil einige theoretische, vor allem aber allgemein praktische Hinweise zur Dünnschicht-Chromatographie. Die handelsüblichen Geräte werden beschrieben; es werden aber auch Angaben gemacht, wie einfache aber ausreichende Behelfe selbst improvisiert werden können. Sodann findet man in dem Buch eine Fülle von Vorschriften zur Dünnschicht-Chromato- graphie verschiedenster Substanzklassen, soz.B. Alkaloiden, Aminosäuren, Steroiden, Lipoiden, Vitaminen, Arzneimittel, Zuckern, Nucleotiden usw. usw. Die Rf-Werte der Stoffe für verschiedene Lösungsmittelsysteme und Schichtmaterialien werden angegeben, die Nachweisverfahren und soweit möglich die quantitative Bestimmung beschrieben. Es handelt sich bei dem Buch von Randerath also um ein ungemein brauchbares Laborbuch, daß bei einem erstaunlich niedrigen Preis eine Fülle von bemerkenswerten Angaben und Vorschriften zugänglich macht. $\mathrm{Da}$ das Buch bei Chemikern, Biochemikern, Pharmakologen, Pharmazeuten, vor allem aber auch im klinisch-chemischen Laboratorium sicher sehr schnell und sehr begeistert aufgenommen wird, ist mit einer baldigen neuen Auflage zu rechnen. Dies ist deshalb so wünschenswert, weil auf dem Gebiet laufend neue Methoden beschrieben werden, die dann bei einer neuen Auflage schnell berücksichtige werden können. Das Buch ist in Druck und Abbildungen bestens ausgestattet.

(Staudinger, Gießen)

Die Wicdergabe von Gebraucbsnamen, Handelsmamen, Warenbezeicbnumgen und dgl. in dieser Zeitscbrift berecbtigt nicbt zu der Annabme, daß solsbe Namen obne visiteres von jedermann benützt werden dürften. Vielmebr bandels es sicb bäufig um gesetzlisb gossbükzte Warenzeicben, aucb wenn sie nicbt aigens als solcbo gekennzeicbnet sind.

Verlag: Walter de Gruyter \& Co., vormals G. J. Göschen'sche Verlagshandlung - J. Guttentag, Verlagsbuchbandlung - Georg Reimer - Karl J. Trübner - Veit \& Comp. 1 Berlin 30, Genthiner Str. 13; 1963. - Printed in Germany. - Satz und Druck: Walter de Gruyter \& Co., 1 Berlin 30, Genthiner Str. 13. - Anzeigen: Merkur-Werbung, Dr. K. Jeserich KG, 7 Stuttgart, 1, Postfach 740, Tel. 246358/59/50. - Für den Anzeigenteil verantwortlich: Willibald Plitzko. 